\title{
Infrared spectroscopy of the 1999 outburst of U Scorpii
}

\author{
A. Evans ${ }^{1}$, J. Krautter ${ }^{2}$, L. Vanzi ${ }^{3}$, and S. Starrfield ${ }^{4}$ \\ 1 Department of Physics, Keele University, Keele, Staffs, ST5 5BG, UK \\ 2 Landessternwarte, Königstuhl, 69117 Heidelberg, Germany \\ e-mail: J.Krautter@lsw. uni-heidelberg. de \\ 3 European Southern Observatory, Casilla 19001, Santiago 19, Chile \\ e-mail: lvanzi@eso.org \\ 4 Department of Physics \& Astronomy, Arizona State University, PO Box 871504, Tempe, AZ 85287-1504, USA \\ e-mail: sumner.starrfield@asu.edu
}

Received 11 December 2000 / Accepted 9 August 2001

\begin{abstract}
We present infrared spectroscopy of the recurrent nova U Sco, obtained during the 1999 eruption. The $0.94 \ldots 2.4 \mu \mathrm{m}$ spectrum is dominated by H I, He I and He II emission lines and the pseudophotosphere of the hot white dwarf. The ejected material is overabundant in $\mathrm{He}$ and $\mathrm{O}$; we estimate $\mathrm{He} / \mathrm{H} \simeq 4.5 \pm 1.7$ and $\mathrm{O} / \mathrm{H} \simeq 0.013$ by number, for electron temperature $2 \times 10^{4} \mathrm{~K}$. There appears to be excess emission longward of $\sim 1.4 \mu \mathrm{m}$, which is likely to be due to free-free emission by the material ejected in the 1999 eruption; from this we deduce an ejected mass of $2.7( \pm 0.9) \times 10^{-7} M_{\odot}$.
\end{abstract}

Key words. stars: individual: U Sco - stars: cataclysmic variables - infrared: stars

\section{Introduction}

$\mathrm{U}$ Sco is a member of the recurrent nova $(\mathrm{RN})$ class of cataclysmic variables, and has undergone eruptions in 1863, 1906, 1936, 1979 and 1987. The "speed" of the eruption is such that, during eruption, the time taken to decline by $3 \mathrm{mag}\left(t_{3}\right)$ is $\sim 4$ days, making it one of the fastest novae of its type (see Warner 1995). It is very likely therefore that a number of eruptions may have been missed. The spectroscopic development of U Sco during outburst differs significantly from that of classical novae in that forbidden lines are generally absent (e.g. Barlow et al. 1981, hereafter B81; Williams et al. 1981), suggesting a high electron density, $N_{\mathrm{e}} \gtrsim 10^{8} \mathrm{~cm}^{-3}$; furthermore, the constant luminosity phase, an essential feature of classical novae, is also apparently absent in U Sco (Williams et al. 1981). There are also significant differences between the spectroscopic development of U Sco and that of other RNe (B81).

Williams et al. (1981) argued that the ejected mass is difficult to determine from $\mathrm{H}$ recombination lines as there are several unknowns, such as the volume filling factor etc. They used instead the width of the absorption in the CIV resonance line to find an ejected mass

Send offprint requests to: A. Evans,

e-mail: ae@astro.keele.ac.uk

* Based on observations obtained at the New Technology Telescope, European Southern Observatory, La Silla, Chile (Target of opportunity observations). of $\sim 10^{-7} M_{\odot}$, substantially less than that typical for classical novae $\left(\sim 10^{-5} M_{\odot}\right)$.

$\mathrm{U}$ Sco is an eclipsing binary with period 1.23056 days (Schaefer \& Ringwald 1995 and references therein). Optical He II lines are detected in emission during quiescence (Johnston \& Kulkarni 1992). These are attributed by Johnston \& Kulkarni to emission by a corona associated with the secondary rather than by a disc; Warner (1995) on the other hand suggests that the primary, heated by RN eruptions, is extremely hot and irradiates the disc to such an extent that the $\mathrm{H}$ is fully ionized and only He II emission is possible.

The spectral type of the secondary is estimated to be F8 \pm 2 by Johnston \& Kulkarni (1992), while a K2 subgiant secondary is deduced by Anupama \& Dewangan (2000; hereafter AD2000); the sub-giant nature of the secondary is also suggested by the orbital period. The mass of the white dwarf (WD) in the U Sco system has been discussed by several authors. Johnston \& Kulkarni (1992) argue for a mass $\sim 0.3 M_{\odot}$, but the consensus is for a nearChandrasekhar mass (Starrfield et al. 1985; Lépine et al. 1999, hereafter LSLZ; Kahabka et al. 1999). Recently, Thoroughgood et al. (2001) obtained optical spectroscopy around an orbit as U Sco returned to minimum light following the 1999 eruption; they deduce a WD mass of $1.55 \pm 0.24 M_{\odot}$, and confirm the evolved nature of the secondary. Models for the outburst of U Sco based on thermonuclear runaway (TNR) on a massive WD have been 


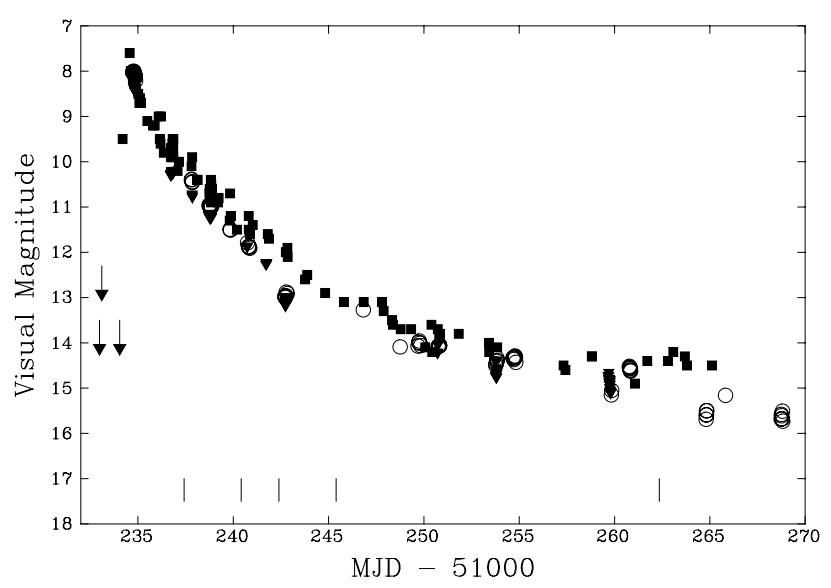

Fig. 1. Visual light curve of U Sco during the 1999 eruption; data from VSNET

(URL: http://www.kusastro.kyoto-u.ac.jp/vsnet/Novae/ usco.html). Filled squares: visual data; open circles: clear filter; filled triangles: $V$ filter; arrows are upper limits. Vertical lines indicate times of IR spectra presented here.

discussed by Truran et al. (1988) and by Starrfield et al. (1985, 1988), who suggest that U Sco is on its way to becoming a Type I supernova.

The distance of U Sco is not well determined, and estimates range from $4 \ldots 14 \mathrm{kpc}$ (see e.g. Hachisu et al. 2000 and references therein). B81 deduced a reddening of $E(B-V) \simeq 0.2 \ldots 0.3$ using several independent techniques, including ultraviolet-optical flux distribution, and H I emission line ratios. A much larger value $(E(B-V) \simeq$ 0.56 ) is deduced by Hachisu et al. (2000) on the basis of their theoretical quiescent light curve. Since the B81 method is based on independent observational estimates we assume the lower value $(E(B-V)=0.2)$ here; the corresponding distance $D=14 \mathrm{kpc}$ (cf. B81, AD2000).

In view of the rarity (relative to the eruptions of classical novae) of RN eruptions, there have been few infrared (IR) spectroscopic observations of RN eruptions. IR spectroscopy of the 1985 outburst of the RN RS Oph - which has a giant secondary - was reported by Evans et al. (1988). They found first-overtone ${ }^{12} \mathrm{CO}$ and ${ }^{13} \mathrm{CO}$ absorption in the secondary and determined that the ${ }^{12} \mathrm{C} /{ }^{13} \mathrm{C}$ ratio was $\sim 10$.

\section{The 1999 outburst}

The most recent outburst of U Sco was discovered by Schmeer (1999) and the visual light curve is shown in Fig. 1. The photometric evolution is discussed by Munari et al. (1999, hereafter MZT), who show that it follows that of previous outbursts to such an extent that the overlaid light curves of the 1979, 1987 and 1999 outbursts are almost indistinguishable. The speed of the 1999 outburst is given by MZT as $t_{2}=2.2$ days and $t_{3}=4.3$ days, and the time of maximum (which we take as the time origin) as $\mathrm{JD}=2451235.062$.
Table 1. Observation log. $m_{\text {vis }}$ are visual magnitude estimates.

\begin{tabular}{ccccc}
\hline Date & MJD & $t$ (days) & $\begin{array}{c}\text { Standard } \\
\text { Star }\end{array}$ & $m_{\text {vis }}$ \\
\hline 1999 Feb. 28 & 51237.41 & 2.34 & HD 151003 & 9.6 \\
1999 Mar. 03 & 51240.39 & 5.33 & HD 153276 & 11.3 \\
1999 Mar. 05 & 51242.37 & 7.21 & HD 125241 & 11.7 \\
1999 Mar. 08 & 51245.39 & 10.37 & HD 125241 & 12.9 \\
1999 Mar. 24 & 51262.34 & 27.28 & HD 151003 & 14.4 \\
\hline
\end{tabular}

Optical spectroscopy is described by MZT, by AD2000 and by Iijima (2001). They find broad emission lines, with the $\mathrm{H} \alpha F W Z I \simeq 10000 \mathrm{~km} \mathrm{~s}^{-1}$, declining to $\simeq 4000 \mathrm{~km} \mathrm{~s}^{-1}$ over the first $\sim 20$ days of the eruption. It is of interest to note that the He II $\lambda 4686$ line was not present on 26 February 1999 (although it was present in 1999 March; AD2000), whereas He II is certainly present in our IR spectra on 28 February (see below), indicating an increase in the degree of ionization in two days. MZT also find that the degree of ionization increases during optical decline, and they suggest that little or no matter is ejected in the eruption, in agreement with Williams et al.'s (1981) conclusion for the 1979 eruption.

LSLZ report HST observations of the $\mathrm{H} \alpha$ line 5 days after the start of the outburst; these authors find an emission component that moves across the spectrum, suggesting acceleration of a clump of material in a moderately collimated outflow. The H I lines initially had an asymmetric saddle profile, later ( $t \gtrsim 18$ days) evolving to a triple peaked profile (MZT, LSLZ).

Kahabka et al. (1999) report a soft X-ray detection with BeppoSAX, and find that the data are consistent with a hot $\left(\sim 9 \times 10^{5} \mathrm{~K}\right)$ massive $\left(\gtrsim 1.2 M_{\odot}\right)$ WD, enhanced $\mathrm{He}$ and an enhanced $\mathrm{C} / \mathrm{N}$ ratio, suggesting TNR and the activity of the CNO cycle (Starrfield et al. 1985, 1988).

\section{Observations}

We present here IR spectroscopy of U Sco obtained during the 1999 eruption.

The observations were carried out with the ESO instrument SOFI on the New Technology Telescope (NTT). A $\log$ of observations is given in Table 1 in which the MJD is effectively at mid-observation and $t$ is the time since maximum; the times of observation are indicated in Fig. 1. On each occasion a spectrum covering the full range of SOFI (0.943. . 2.55 $\mu \mathrm{m}$; Lidman et al. 1999) was obtained, although the spectra longward of $\sim 2.4 \mu \mathrm{m}$ are generally too noisy to be useful; the spectral resolution $\lambda / \delta \lambda=930$ in the wavelength range $0.95 \ldots 1.64 \mu \mathrm{m}$, and $=980$ in the range $1.53 \ldots 2.52 \mu \mathrm{m}$ (Lidman et al. 1999). All the observations were scheduled to occur outside of eclipse. Sky and telluric subtraction was achieved by nodding the target along the slit, and by observing the stars HD 151003 (O9Iab/Ib, $T_{\text {eff }}=32500 \mathrm{~K}, J=7.73, H=$ $7.78, K=7.91)$, HD 125241 (O9I, $T_{\text {eff }}=32500 \mathrm{~K}$, 


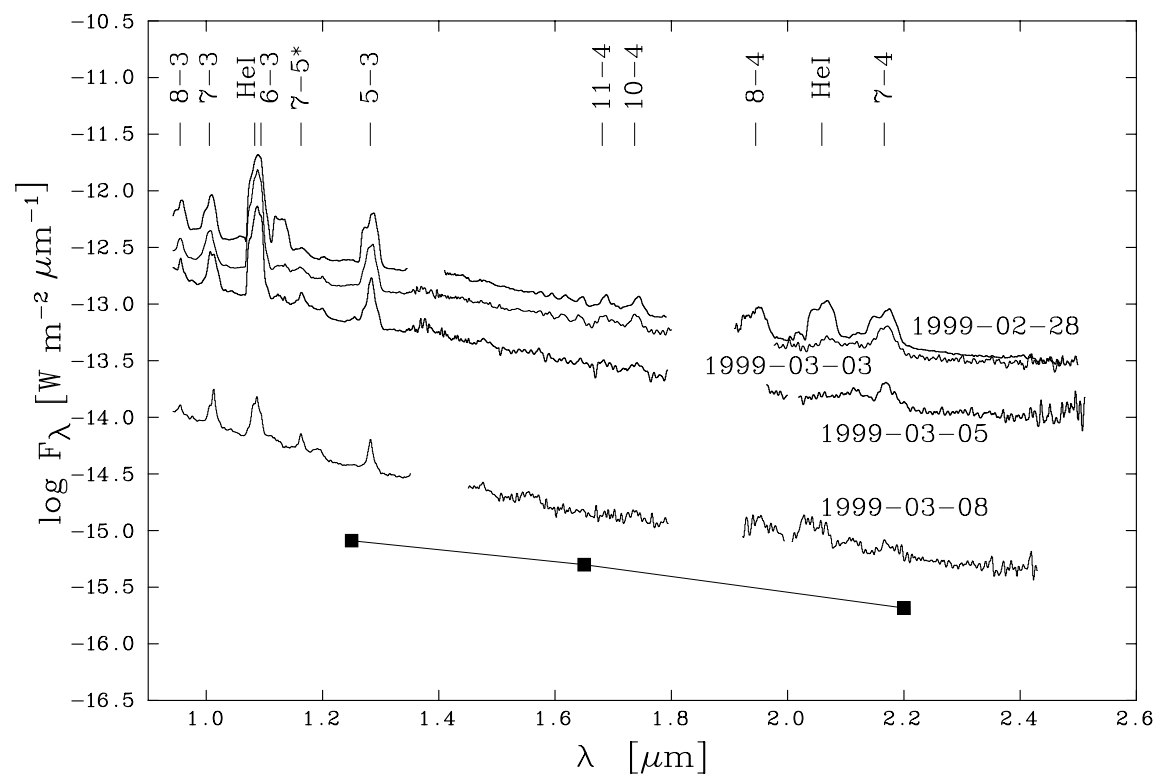

Fig. 2. Evolution of the observed spectrum of U Sco in the $0.95 \ldots 2.4 \mu \mathrm{m}$ range. Line identifications are given along the top of the figure, in the form $M-N$ for H I $M-N+$ He II $2 M-2 N$ transitions; features indicated by $M-N^{*}$ are He II only. The three points at the bottom of the diagram are an estimate of the $J H K$ fluxes of $\mathrm{U}$ Sco at quiescence, reddened by $E(B-V)=0.2$. See text for details.

$J=8.87, H=8.92, K=9.05)$ and $\mathrm{HD} 153276(\mathrm{G} 3 \mathrm{~V}$, $\left.T_{\text {eff }}=5785 \mathrm{~K}, J=7.10, H=6.77, K=6.72\right)$ at the same airmass, where the colours and effective temperatures have been taken from Tokunaga (1998). We interpolated across $H$ lines in the $\mathrm{G}$ star spectrum before flux-calibrating the U Sco observations; the flux calibration is accurate to $\pm 20 \%$. A xenon arc was used for wavelength calibration, which should be accurate to $0.001 \mu \mathrm{m}$; the spectra were smoothed with a $0.001 \mu \mathrm{m}$ filter.

The evolution of the observed spectrum in the $0.95 \ldots 2.5 \mu \mathrm{m}$ range is shown in Fig. 2, which also includes an estimate of the $J H K$ fluxes at quiescence, based on a quiescent visual magnitude $V=17.9$ (Webbink et al. 1987) and a K2IV secondary (AD2000), for which we have taken appropriate IR colours $[(V-K)=2.22,(J-H)=$ $0.50,(H-K)=0.09]$ from Tokunaga (1998); this assumes of course that the visual magnitude is dominated by the secondary. The spectrum obtained on 1999 March 24 shows no secure evidence of emission by U Sco, although there may be very weak He I $\lambda 1.083$ emission (see Fig. 3 ).

\section{Discussion}

\subsection{The continuum}

In Fig. 4 we show our IR spectra, together with the visual estimates at the time of our observations (see Table 1); the data have been dereddened by $E(B-V)=0.2$ (see Sect. 1).

Visual estimates differ from $V$ magnitudes by an amount that depends on $(B-V)$ and on the (unknown and almost certainly variable) zero point of the visual magnitude scale (see Bailey \& Howarth 1979; Howarth \& Bailey 1980 for detailed discussion). During the 1979 eruption,

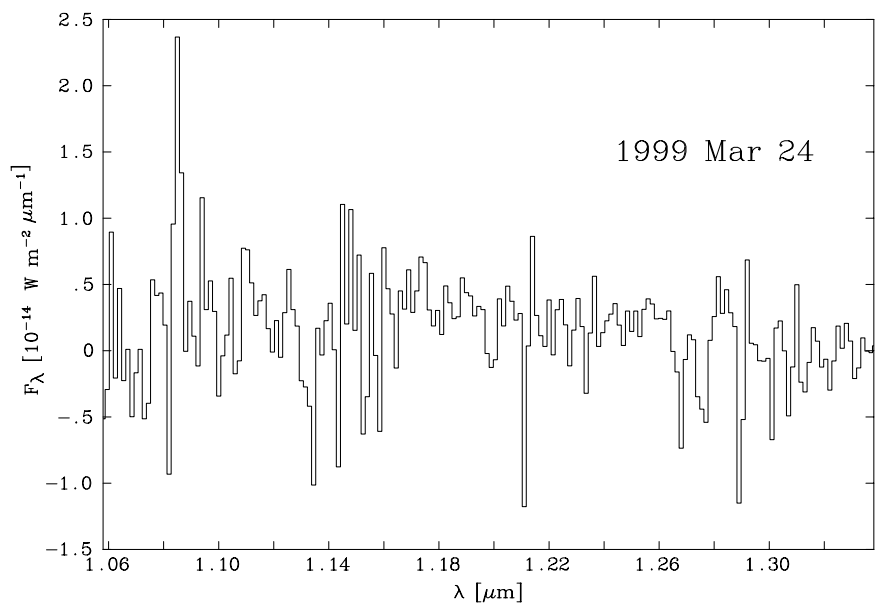

Fig. 3. The observed spectrum in the region of the He I $\lambda 1.083$ line on 1999 March 24.

Table 2. Estimates of the photospheric temperature $T_{\mathrm{pp}}$ of the WD pseudophotosphere.

\begin{tabular}{ccc}
\hline Date & $t$ (days) & $T_{\mathrm{pp}}(1000 \mathrm{~K})$ \\
\hline 1999 Feb. 28 & 2.34 & 30 \\
1999 Mar. 03 & 5.33 & 10 \\
1999 Mar. 05 & 7.21 & $10 \ldots 15$ \\
1999 Mar. 08 & 10.37 & $\gtrsim 50$ \\
\hline
\end{tabular}

the $(B-V)$ colour of $\mathrm{U}$ Sco varied between -0.13 and -0.28 and, on average, became bluer as the eruption progressed (B81). As noted in Sect. 2, the visual light curve of U Sco is essentially indistinguishable from outburst to outburst. If we assume that the colour evolution is likewise 

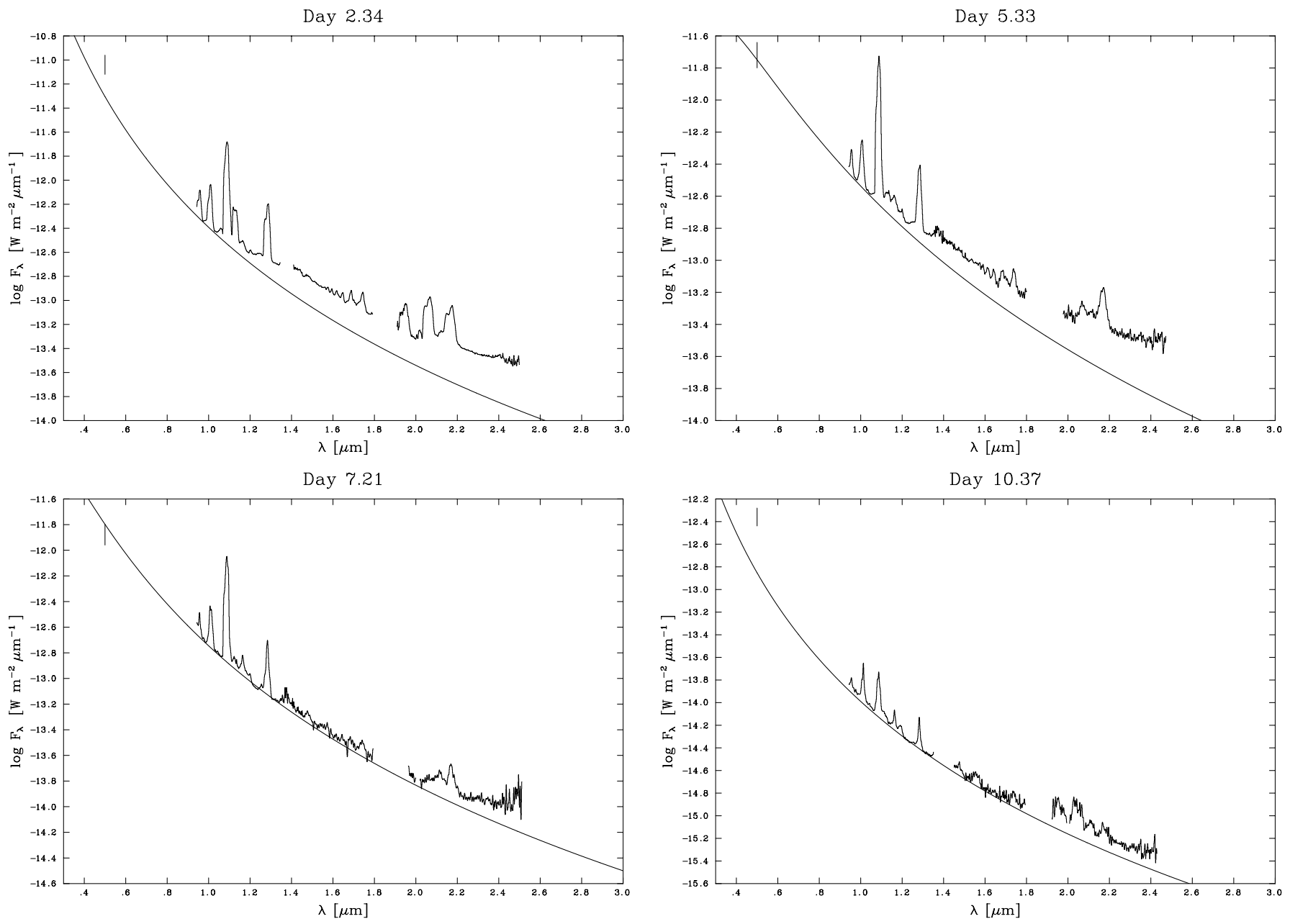

Fig. 4. Optical ( $V$-band) IR flux distribution of U Sco for the four epochs shown. Vertical ticks at $\lambda=0.50 \mu \mathrm{m}$ are visual eye estimates, with an allowance of \pm 0.2 for uncertainties in interpolation and in the eye estimates. Curves are blackbody fits to the optical/IR data. See text and Table 2 for details.

similar, we can use the prescription in Bailey \& Howarth (1979) to estimate the $V$ magnitude. Since U Sco is relatively blue during outburst (B81), the $V$ magnitude is fainter than the corresponding visual estimates (Howarth \& Bailey 1980); this effect can clearly be seen in Fig. 1. The correction is typically $\simeq 0.05$ mag but as noted above, this does not include the unknown contribution from errors in the zero point.

We also note that the effective wavelength of the darkadapted eye is not well-known; it will vary from observer to observer, and will almost certainly change as the nova evolves. It is known to be blueward of the standard $V$ filter (e.g. Allen 1973) and we assume here a value $0.50 \mu \mathrm{m}$. The resulting approximate $V$ magnitudes are included in Fig. 4 and, in view of the uncertainties, they should be taken only as a guide to the behaviour of the flux distribution at shorter wavelengths.

We use our IR spectra in an attempt to constrain the temperature of the WD pseudophotosphere, which we approximate by a black body at temperature $T_{\mathrm{pp}}$. While the $T_{\mathrm{pp}}$ values are not well constrained by our data (see Table 2 and Fig. 4), we obtain values in the range $10^{4} \ldots 3 \times 10^{4} \mathrm{~K}$ over the first $\sim 7$ days, and
$T_{\mathrm{pp}} \gtrsim 50 \times 10^{3} \mathrm{~K}$ for day 10.37 ; values $\lesssim 10^{4} \mathrm{~K}$ are excluded and the uncertainty is typically $\pm 3000 \mathrm{~K}$. A similar range of values is obtained if we use a reddening of $E(B-V)=0.6($ see Sect. 1$)$.

We note that, for two datasets (Day 2.34 and Day 10.37), the visual estimates lie well above the extrapolated black body (see Fig. 4) and there is no reasonable value of $E(B-V)$ or $T_{\mathrm{pp}}$ that renders the visual data and our IR spectra consistent with black body emission. Noting the caveat above regarding the visual magnitudes, the likely explanation for this discrepancy is that the WD pseudophotosphere is not well-described by a black body over the wavelength range $0.50 \ldots 2.5 \mu \mathrm{m}$. An alternative, though less likely, explanation is the presence of strong emission lines around $5000 \AA$ where the sensitivity of the eye peaks (Allen 1973); however this may not apply in the case of U Sco as, with the exception of He II $\lambda 4686$ in March 1999 (AD2000), there were in general no strong lines (such as [O III]) in this wavelength region in either the 1979 (B81) or the 1999 (AD2000) eruptions. We note however that the optical spectra presented by AD2000 for $t=1.45$ days and $t=11.41$ days lie close to our blackbody curves for days 2.34 and 10.37 . 
As is evident from Fig. 2, at no time do we see the underlying system in our data. However there seems to be an excess relative to the WD pseudophotosphere for wavelengths $\gtrsim 1.4 \mu \mathrm{m}$, amounting to $\sim 10^{-14} \mathrm{~W} \mathrm{~m}^{-2} \mu \mathrm{m}^{-1}$ on day 2.34 and declining to $\sim 10^{-16} \mathrm{~W} \mathrm{~m}^{-2} \mu \mathrm{m}^{-1}$ by day 10.37 (see Fig. 4). This excess is too large to be due to, for example, uncertainty in the temperature of the standard stars or in the reddening. We discuss this excess further in Sect. 4.2.4 below.

\subsection{The emission lines}

In general, the IR emission lines show the characteristic asymmetric structure seen in the optical (MZT, LSLZ), with the red component stronger than the blue component. Most of the lines are identified with $\mathrm{HI}$, He I and He II; the Br lines up to the 16-4 line are clearly visible in the data. The profiles of He I, Br $\gamma+\mathrm{He}$ II $14-8$ and $\mathrm{Pa} \beta+\mathrm{He}$ II $10-6$ are shown in Fig. 5a, which emphasizes the similarity between the profiles of the He I and the $\mathrm{H} \mathrm{I}+\mathrm{He}$ II recombination lines. We note that, at the resolution of our spectra, and the velocities implied by the line widths (see Fig. 5a), the $1.0833 \mu \mathrm{m}$ line is blended with Pa $\gamma+\mathrm{He}$ II $12-6$ at $1.093 \mu \mathrm{m}$, which accounts for the apparent greater width of the He I line in Fig. 5a.

The fluxes, central wavelengths and $F W H M$ for the stronger emission lines have been determined by fitting two Gaussians to the dereddened line profiles. The central wavelengths, line widths and fluxes as determined from the dereddened spectra are listed in Table 3. The formal errors in the line fluxes are typically $\pm 5 \%$ for the stronger lines, and typically $\pm 15 \%$ for the weaker; within a given wavelength band the flux ratios will therefore have a typical $7 . .20 \%$ error.

The velocities $V_{1}$ and $V_{3}$ are heliocentric and follow from the observed wavelengths of the red- and blueshifted line components respectively, while $V_{2}$ and $V_{4}$ are determined from the respective $F W H M$; the instrumental contribution has been removed from the measured $F W H M$ values, but no attempt has been made to decon-

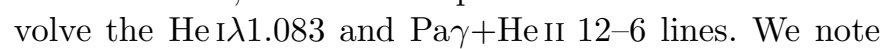
that the FWZI $\left(\sim 10000 \ldots 12000 \mathrm{~km} \mathrm{~s}^{-1}\right.$; see Fig. 5) for He I $\lambda 1.083$ is comparable with - but somewhat greater than - the value determined for $\mathrm{H} \alpha$ (MZT) over the first 10 days of the eruption $\left(\sim 9000 \ldots 10000 \mathrm{~km} \mathrm{~s}^{-1}\right)$, no doubt because of the blending of the HeI line with Pa + He II 12-6 (see above). The FWZI as determined from the $\mathrm{He}$ I $\lambda 2.058$ line is more in line with the $\mathrm{H} \alpha$ data. Indeed the $F W Z I$ of the HeI $\lambda 2.058$ line $\left(9200 \mathrm{~km} \mathrm{~s}^{-1}\right)$ is close to the value predicted by Eq. (3) of MZT $\left(9400 \mathrm{~km} \mathrm{~s}^{-1}\right)$ for the $F W Z I$ of $\mathrm{H} \alpha$ at $t=2.34$ days. We note that the wavelength difference between H I $M-N$ lines and the corresponding He II $2 M-2 N$ lines corresponds to a velocity difference of $\sim 120 \mathrm{~km} \mathrm{~s}^{-1}$; these lines are not therefore resolved in our data.

On 1999 February 28, on which we have several lines to provide reasonable statistics, the mean velocities for the $\mathrm{HI}$ and HeI $\lambda 2.06$ lines are $\overline{V_{1}}=1083 \pm 53 \mathrm{~km} \mathrm{~s}^{-1}$, $\overline{V_{3}}=-2628 \pm 140 \mathrm{~km} \mathrm{~s}^{-1}, \overline{V_{2}}=2020 \pm 141 \mathrm{~km} \mathrm{~s}^{-1}$ and $\overline{V_{4}}=1238 \pm 77 \mathrm{~km} \mathrm{~s}^{-1}$. The mean value of the $R / B$ ratio is $3.91 \pm 0.35$, where the quoted errors are the standard errors of the means.

Coronal lines are frequently seen in the IR spectra of novae (Greenhouse et al. 1990) and were present in the IR spectrum of RS Oph during the 1985 eruption (Evans et al. 1988); however they were not seen in the IR spectrum of U Sco. The implication is that the strong interaction between the material ejected in the 1985 eruption of RS Oph and the giant secondary wind did not occur in the case of U Sco; this is no doubt a consequence of the different environment in the two cases: presumably there is no substantial wind from the secondary star in the case of U Sco. It is also noteworthy that there were no forbidden lines in either the IR or the optical (AD2000) spectra.

\subsubsection{The $\mathrm{He}$ I lines}

The fluxes, central wavelengths and $F W H M$ for the He I lines at $1.0833 \mu \mathrm{m}\left(3 \mathrm{~S}-3 \mathrm{P}^{\circ}\right)$ and at $2.0587 \mu \mathrm{m}\left(1 \mathrm{~S}-1 \mathrm{P}^{\circ}\right)$ lines are listed in Table 3 ; the fluxes are largely confined to the stronger $1.083 \mu \mathrm{m}$ line which, as already noted, is contaminated by $\mathrm{P} \gamma+\mathrm{He}$ II $12-6$; the $\lambda 2.058$ line is too weak after the first observation to measure meaningful velocities.

The evolution of the HeI $\lambda 1.083$ profile is shown in Fig. 5b. These profiles bear a very strong resemblance to the $\mathrm{H} \alpha$ profiles presented by MZT and by LSLZ, indicating that $\mathrm{He} \mathrm{I}$ and $\mathrm{H}$ are similarly distributed in the emitting material (or of course that there is negligible contribution from $\mathrm{H}$ I recombination lines). By $t=7.21$ days we begin to see evidence for a shoulder on the red wing of He I $\lambda 1.083$ (see Fig. 5b), possibly as a result of the declining velocities reported by MZT and the emergence of Pa $\gamma+$ He II 12-6; the corresponding $V$ values in Table 3, particularly for day 10.37 , should therefore be regarded with caution.

\subsection{2. $\mathrm{H}-\mathrm{He}$ recombination lines}

The majority of the other emission lines in Fig. 2 can be attributed to $\mathrm{H}$ I+He II recombination lines.

In principle we can use the $\mathrm{HI}+\mathrm{He}$ II recombination lines to determine the $\mathrm{He} / \mathrm{H}$ ratio in the emitting material. Unfortunately however the flux ratios for these lines are insensitive to the electron density $N_{\mathrm{e}}$, the electron temperature $T_{\mathrm{e}}$ and the $\mathrm{He} / \mathrm{H}$ ratio. However we can use the weaker He II line at $1.164 \mu \mathrm{m}$, to which the only contribution is from He II $7-5(\lambda=1.16296 \mu \mathrm{m})$ and He II $11-6$ $(\lambda=1.16764 \mu \mathrm{m})$. The flux ratio gives $n\left(\mathrm{He}^{++}\right) / n\left(\mathrm{H}^{+}\right)$, from which the $\mathrm{He} / \mathrm{H}$ ratio follows if it assumed that $\mathrm{H}$ and He are the only sources of electrons and that the distribution of ionization states is given by the Saha equation. The dereddened flux ratios He II/H I for the He II 7-5 and 
Table 3. Central wavelengths and line widths for stronger emission lines. Spectra have been dereddened for $E(B-V)=0.2$. Fluxes are written in the form $X(-Y)=X \times 10^{-Y}$. All H I $M-N$ recombination lines have a contribution from the corresponding He II $2 M-2 N$ lines. $\mathrm{R} / \mathrm{B}$ is the ratio of the fluxes in the red and blue components. See text for details.

\begin{tabular}{|c|c|c|c|c|c|c|c|c|c|c|c|}
\hline \multirow{2}{*}{\multicolumn{2}{|c|}{ Date }} & \multirow{2}{*}{$\begin{array}{c}t \\
\text { (days) }\end{array}$} & \multirow{2}{*}{$\begin{array}{l}\lambda_{\mathrm{obs}} \\
(\mu \mathrm{m})\end{array}$} & \multirow[t]{2}{*}{ ID } & \multirow{2}{*}{$\begin{array}{c}F W H M \\
(\mu \mathrm{m})\end{array}$} & \multirow{2}{*}{$\begin{array}{l}\text { Line flux } \\
\left(\mathrm{W} \mathrm{m}^{-2}\right)\end{array}$} & $V_{1}$ & $V_{2}$ & $V_{3}$ & $V_{4}$ & \multirow[t]{2}{*}{$R / B$} \\
\hline & & & & & & & \multicolumn{4}{|c|}{$\left(\mathrm{km} \mathrm{s}^{-1}\right)$} & \\
\hline \multirow{20}{*}{\multicolumn{2}{|c|}{1999 Feb. 28}} & 2.34 & 1.009 & $\mathrm{P} \delta($ Н г 7-3) & 0.0147 & $8.24(-15)$ & 1163 & 2162 & & & 5.77 \\
\hline & & & 0.996 & & 0.0085 & $1.43(-15)$ & & & -2687 & 1230 & \\
\hline & & & 1.089 & Не I $\lambda 1.08$ & 0.0192 & $3.70(-14)$ & 1524 & 2620 & & & 9.66 \\
\hline & & & 1.075 & & 0.0081 & $3.83(-15)$ & & & -2322 & 1077 & \\
\hline & & & 1.165 & Не II $7-5,11-6$ & 0.0153 & $7.63(-16)$ & 453 & 1944 & & & 4.78 \\
\hline & & & 1.153 & & 0.0076 & $1.60(-16)$ & & & -2537 & 933 & \\
\hline & & & 1.132 & О г $3 \mathrm{P}-3 \mathrm{D}^{\circ}, 5 \mathrm{P}-5 \mathrm{~S}^{\circ} ?$ & 0.0161 & $4.98(-15)$ & 760 & 2139 & & & 2.08 \\
\hline & & & 1.119 & & 0.0075 & $2.40(-15)$ & & & -2770 & 950 & \\
\hline & & & 1.287 & $\mathrm{P} \beta\left(\mathrm{H}_{\text {I }} 5-3\right)$ & 0.0173 & $8.02(-15)$ & 1045 & 1986 & & & 3.71 \\
\hline & & & 1.271 & & 0.0093 & $2.16(-15)$ & & & -2626 & 1053 & \\
\hline & & & 1.688 & $\mathrm{Br} \eta(\mathrm{H}$ I 11-4) & 0.0146 & $5.38(-16)$ & 1241 & 1255 & & & 2.87 \\
\hline & & & 1.670 & & 0.0188 & $1.88(-16)$ & & & -1880 & 1622 & \\
\hline & & & 1.743 & $\operatorname{Br} \zeta($ H I 10-4) & 0.0223 & $9.52(-16)$ & 1137 & 1889 & & & 3.90 \\
\hline & & & 1.720 & & 0.0161 & $2.44(-16)$ & & & -2885 & 1366 & \\
\hline & & & 1.951 & $\operatorname{Br} \delta\left(\mathrm{H} \mathrm{I}_{8-4}\right)$ & 0.0290 & $1.41(-15)$ & 941 & 2207 & & & 3.99 \\
\hline & & & 1.926 & & 0.0148 & $3.53(-16)$ & & & -2897 & 1113 & \\
\hline & & & 2.067 & Не I $\lambda 2.06$ & 0.0327 & $2.28(-15)$ & 1193 & 2349 & & & 3.93 \\
\hline & & & 2.042 & & 0.0154 & $5.80(-16)$ & & & -2475 & 1056 & \\
\hline & & & 2.172 & $\operatorname{Br} \gamma\left(\mathrm{H}_{\text {I }} 7-4\right)$ & 0.0335 & $1.81(-15)$ & 861 & 2290 & & & 3.19 \\
\hline & & & 2.145 & & 0.0181 & $5.68(-16)$ & & & -2945 & 1227 & \\
\hline \multirow{10}{*}{\multicolumn{2}{|c|}{1999 Mar. 03}} & 5.33 & 1.007 & $\mathrm{P} \delta($ Н г 7-3) & 0.0142 & $3.71(-15)$ & 591 & 2089 & & & 4.72 \\
\hline & & & 0.998 & & 0.0131 & $7.89(-16)$ & & & -2210 & 1949 & \\
\hline & & & 1.088 & Не I $\lambda 1.08$ & 0.0174 & $3.01(-14)$ & 1220 & 2560 & & & 13.6 \\
\hline & & & 1.073 & & 0.0060 & $2.21(-15)$ & & & -2440 & 826 & \\
\hline & & & 1.162 & Не ІІ $7-5,11-6$ & 0.0179 & $8.17(-16)$ & -243 & 2283 & & & 5.34 \\
\hline & & & 1.148 & & 0.0089 & $1.53(-16)$ & & & -3904 & 1123 & \\
\hline & & & 1.285 & $\mathrm{P} \beta\left(\mathrm{H} \mathrm{I}^{5-3)}\right.$ & 0.0149 & $3.57(-15)$ & 694 & 1704 & & & 4.14 \\
\hline & & & 1.274 & & 0.0117 & $8.61(-16)$ & & & -1885 & 1335 & \\
\hline & & & 2.170 & $\operatorname{Br} \gamma\left(\mathrm{H}_{\text {I }} 7-4\right)$ & 0.0288 & $8.82(-16)$ & 529 & 1974 & & & 15.34 \\
\hline & & & 2.157 & & 0.0090 & $5.75(-17)$ & & & -1257 & 542 & \\
\hline \multirow{8}{*}{\multicolumn{2}{|c|}{1999 Mar. 05}} & 7.21 & 1.012 & $\mathrm{P} \delta\left(\mathrm{H}_{\text {I }} 7-3\right)$ & 0.0143 & $2.00(-15)$ & 1998 & 2087 & & & 5.53 \\
\hline & & & 1.005 & & 0.0064 & $3.61(-16)$ & & & -30 & 891 & \\
\hline & & & 1.087 & Не I $\lambda 1.08$ & 0.0150 & $9.76(-15)$ & 1137 & 2041 & & & 7.39 \\
\hline & & & 1.074 & & 0.0070 & $1.32(-15)$ & & & -2461 & 926 & \\
\hline & & & 1.164 & Не II $7-5,11-6$ & 0.0095 & $3.13(-16)$ & 298 & 1181 & & & 7.36 \\
\hline & & & 1.154 & & 0.0058 & $4.25(-17)$ & & & -2228 & 675 & \\
\hline & & & 1.284 & $\mathrm{P} \beta\left(\mathrm{H}_{\text {I }} 5-3\right)$ & 0.0147 & $1.56(-15)$ & 337 & 1629 & & & 11.93 \\
\hline & & & 1.269 & & 0.0087 & $1.31(-16)$ & & & -3071 & 978 & \\
\hline \multirow{6}{*}{\multicolumn{2}{|c|}{1999 Mar. 08}} & 10.37 & 1.013 & $\mathrm{P} \delta\left(\mathrm{H}_{\text {I }} 7-3\right)$ & 0.0078 & $8.62(-17)$ & 2201 & 1102 & & & 3.91 \\
\hline & & & 1.005 & & 0.0055 & $2.20(-17)$ & & & -2117 & 756 & \\
\hline & & & 1.087 & Не I $\lambda 1.08$ & 0.0091 & $1.01(-16)$ & 999 & 1212 & & & 2.51 \\
\hline & & & 1.079 & & 0.0074 & $4.03(-17)$ & & & -1243 & 982 & \\
\hline & & & 1.163 & Не ІІ $7-5,11-6$ & 0.0099 & $2.29(-17)$ & - & - & - & - & - \\
\hline & & & 1.282 & $\mathrm{P} \beta($ Н I $5-3)$ & 0.0112 & $3.62(-17)$ & - & - & - & - & - \\
\hline 1999 & Mar. 24 & 27.28 & 1.085 & Не г $\lambda 1.08$ & - & $\lesssim 5(-17)$ & - & - & & & \\
\hline
\end{tabular}



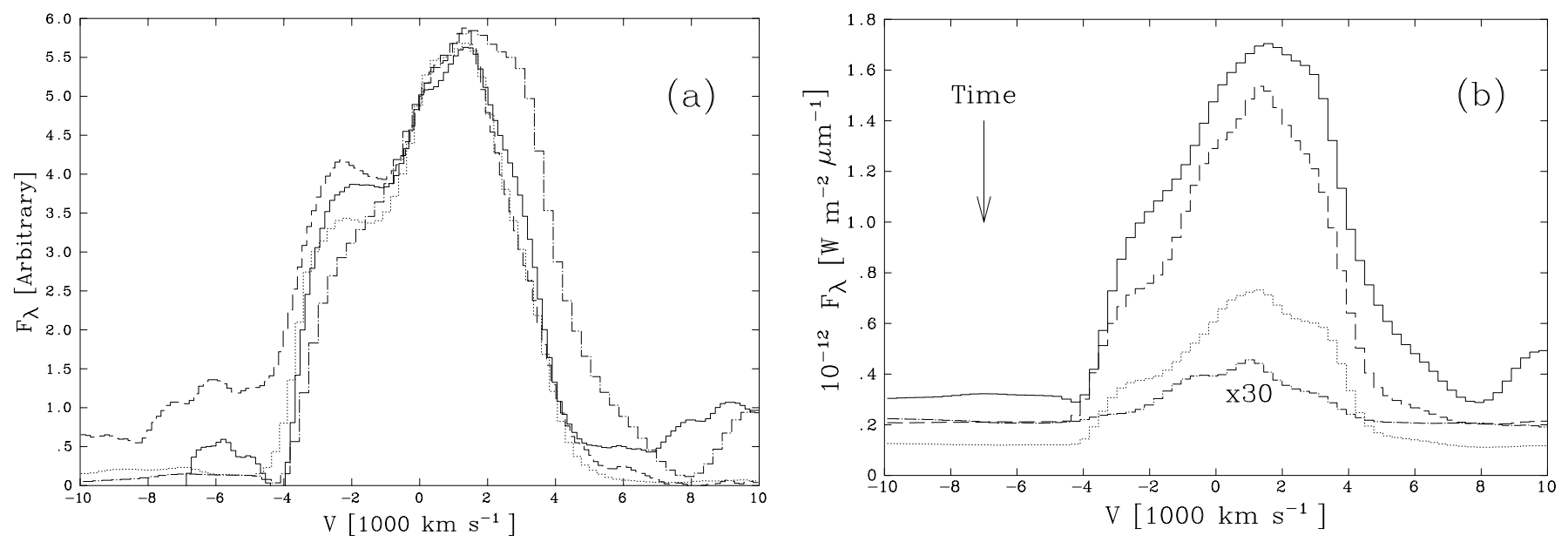

Fig. 5. a) The profiles of He I $\lambda 2.058$ (full curve), Br $\gamma+$ He II $14-8$ (broken curve), Pa $\beta+$ He II $10-6$ (dotted curve) and He I $\lambda 1.083$ (dash-dot curve) on 1999 February 28. The profiles have been normalized at maximum flux; vertical scale is arbitrary. b) Observed profile of the HeI $\lambda 1.083$ line in velocity space for 1999 Feb. 28 (full curve), 1999 March 3 (broken curve), 1999 March 8 (dotted curve), 1999 March 8 (dash-dot curve); the flux for the 1999 March 8 spectrum has been multiplied by 30.

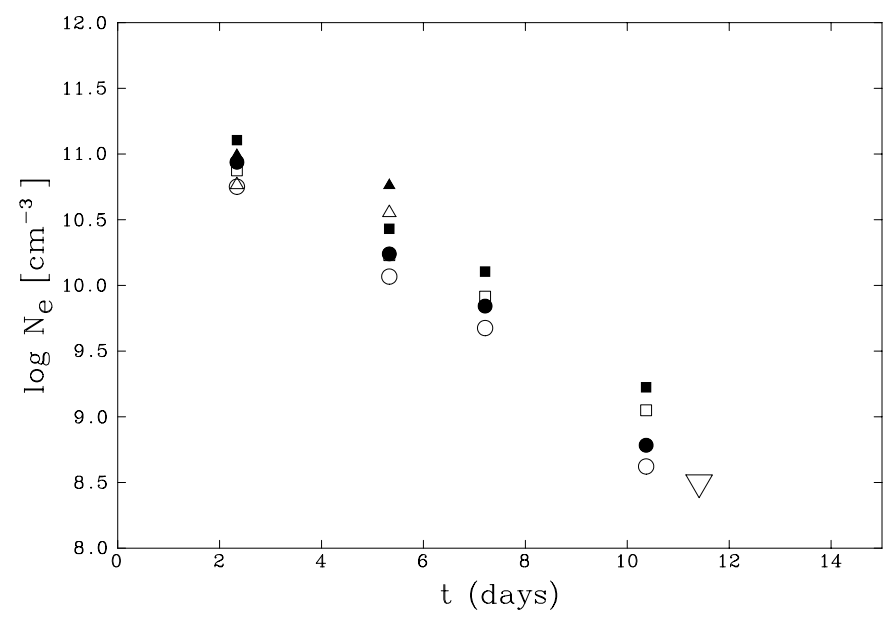

Fig. 6. Dependence of $N_{\mathrm{e}}$ on time since eruption, as determined from the $\mathrm{P} \beta$ (circles), $\mathrm{P} \delta$ (squares) and $\mathrm{Br} \gamma$ (triangles) lines. Filled symbols, $T_{\mathrm{e}}=10^{4} \mathrm{~K}$, open symbols, $T_{\mathrm{e}}=2 \times 10^{4} \mathrm{~K}$. The inverted triangle at $t=11.4$ days is the $N_{\mathrm{e}}$ from AD2000, scaled to the $V$ and $\phi$ values used here. See text for details.

H I Pa lines are given in Table 4, in which the fluxes in the red and blue components have been summed.

To estimate the $\mathrm{He} / \mathrm{H}$ ratio we need to determine $N_{\mathrm{e}}$. In principle we can estimate the electron density $N_{\mathrm{e}}$ in two ways:

(i) We first assume that the density declines as $t^{-3}$ and use the fluxes in the IR H I recombination lines (AD2000). The flux is

$$
\begin{aligned}
f(\mathrm{HI}) & =\epsilon N_{\mathrm{e}} N(\mathrm{H})\left(\frac{4 \pi V^{3} t^{3}}{3}\right) \frac{\phi}{4 \pi D^{2}} \\
& =\frac{\epsilon \phi N_{\mathrm{e}}^{2}}{\alpha} \frac{V^{3} t^{3}}{3 D^{2}}
\end{aligned}
$$

where $\epsilon$ is the case B emissivity from Storey \& Hummer (1995) and which is $N_{\mathrm{e}^{-}}$and $T_{\mathrm{e}}$-dependent, $\alpha$ is the number of electrons per $\mathrm{H}$ and depends on the He: $\mathrm{H}$ ratio $y, N_{\mathrm{e}}$ and $T_{\mathrm{e}} ; \phi$ is the volume filling factor. Assuming that the emission is optically thin in the H I 5-3, 7-3 and 7-4 lines, we iterate to determine $N_{\mathrm{e}}$ and find the time-dependence shown in Fig. 6, in which velocity $2000 \mathrm{~km} \mathrm{~s}^{-1}$, filling factor $10^{-2}$ and $\alpha=1$ have been assumed. The same values of $N_{\mathrm{e}}$ of course arise for any combination satisfying $V^{3} \phi / \alpha=8 \times 10^{7} \mathrm{~km}^{3} \mathrm{~s}^{-3}$. Also shown in Fig. 6 is the value of $N_{\mathrm{e}}$ deduced for $t=11.4$ days by AD2000 from the optical H I lines, scaled to the values of $V$ and $\phi$ used here. The values of $N_{\mathrm{e}}$ are in satisfactory agreement. We see that $N_{\mathrm{e}} \simeq 10^{11} \mathrm{~cm}^{-3}$ at the time of our earliest observation, consistent with the absence of forbidden lines in the optical (cf. B81; Williams et al. 1981; AD2000).

(ii) We can also use the expected mass-loss from a massive WD at $T \simeq 9 \times 10^{5} \mathrm{~K}$ (Kahabka et al. 1999), namely $\sim 1.2 \times 10^{-6} M_{\odot} \mathrm{yr}^{-1}$, a value that is consistent with the X-ray emission during the 1999 eruption. Assuming that the only sources of electrons in a steady, spherically symmetric wind are $\mathrm{H}$ and $\mathrm{He}$, the corresponding electron density at time $t$ is

$N_{\mathrm{e}} \simeq \frac{6.04 \times 10^{7}}{t_{\mathrm{d}}^{2} V_{2000}^{3} \phi} \frac{\alpha}{1+4 y} \mathrm{~cm}^{-3}$,

where $t_{\mathrm{d}}$ is in days and $V_{2000}$ is the velocity in units of $2000 \mathrm{kms}^{-1}$. The value of $\alpha /(1+4 y)$ is typically in the range $0.3 \ldots 0.8$ for a wide range of wind parameters. For $\phi \simeq 10^{-2}$ and $\alpha /(1+4 y)=0.6, N_{\mathrm{e}} \simeq$ $6.6 \times 10^{8} \mathrm{~cm}^{-3}$, significantly smaller than the value deduced from the $\mathrm{H}$ I recombination lines but still consistent with the absence of forbidden lines in the optical.

Presumably the difference in $N_{\mathrm{e}}$ values reflects the inhomogeneity of the ejecta and the fact that the optical/IR 
and X-ray observations sample different physical regions of the ejecta. To estimate the $\mathrm{He} / \mathrm{H}$ ratio we use the values of $N_{\mathrm{e}}$ as determined from the recombination lines. The mean values for each day of observation are given in Table 4 , for $T_{\mathrm{e}}=2 \times 10^{4} \mathrm{~K}$ and $\alpha=1$.

The deduced $\mathrm{He} / \mathrm{H}$ ratios $(=y)$ for electron temperature $T_{\mathrm{e}}=2 \times 10^{4} \mathrm{~K}$ are given in Table 4 , in which it is seen that $y$ is significantly greater that the solar value. The mean value is $y=4.5 \pm 1.7$, where the error includes the effect of the scatter in the individual $y$ values, and an uncertainty in flux ratios of $\pm 20 \%$ (see Sect. 4.2). The value of $\alpha$ corresponding to $y=4.5, T_{\mathrm{e}}=2 \times 10^{4} \mathrm{~K}$ and the electron densities in Table 4 is $\simeq 5.5$. The $N_{\mathrm{e}}$ values in Table 4 should in principle therefore be increased by a factor $\sim 2.4$; however in view of the uncertainties in $\phi$ etc., we have not applied this correction.

This estimate of $\mathrm{He} / \mathrm{H}$ is greater than that deduced by B81 for the 1979 eruption $(\mathrm{He} / \mathrm{H} \simeq 2)$, and significantly higher than the value obtained by $\mathrm{AD} 2000(\mathrm{He} / \mathrm{H} \simeq 0.4 \pm$ $0.06)$ and Iijima $(2001 ; 0.16)$ from the strength of optical $\mathrm{H}$ and $\mathrm{He}$ lines in the 1999 eruption. A possible reason for this discrepancy is that AD2000 and Iijima (2001) did not allow for the contribution of He II lines to the Hi lines. A further reason, in terms of the opacity of the ejected material to $\mathrm{H}$ - and He-ionizing photons, is discussed by Iijima (2001). All these methods of course assume that the $\mathrm{H}$ and He occupy the same regions of the ejecta, with identical spatial distribution, and having identical values of $N_{\mathrm{e}}$ and $T_{\mathrm{e}}$. However the similarity of the $\mathrm{H}$ and $\mathrm{He}$ emission line profiles (see Sect. 4.2.1) suggests strongly that this is the indeed the case.

In principle, a limit on $\mathrm{He} / \mathrm{H}$ also follows from the HeI 2.06/Br $\gamma$ ratio (Krautter et al. 1984), although it transpires that the limit is not useful in the case of U Sco. Here we modify the treatment of Krautter et al. (1984) to take into account (a) the contribution of He II 148 to $\operatorname{Br} \gamma$, (b) the more recent emissivities in Storey \& Hummer (1995), and (c) recently determined He II recombination coefficients from Hummer \& Storey (1998). The He I $2.06 / \mathrm{Br} \gamma$ ratio at $t=2.34$ days is $\simeq 1.2$ (see Table 3 ), so that $n\left(\mathrm{He}^{+}\right) / n\left(\mathrm{H}^{+}\right) \gtrsim 0.09$. For $N_{\mathrm{e}} \lesssim 10^{10} \mathrm{~cm}^{-3}$ and $T_{\mathrm{e}} \gtrsim 10^{4} \mathrm{~K}, n\left(\mathrm{He}^{+}\right) / n\left(\mathrm{H}^{+}\right)$is very close to $n(\mathrm{He}) / n(\mathrm{H})$, so that all we can conclude from this method is that $\mathrm{He} / \mathrm{H} \geq 0.09$ by number.

\subsubsection{Other lines}

On 1999 February 28 there is also a strong emission feature at $\lambda \simeq 1.13 \mu \mathrm{m}$, which weakens considerably over the remaining spectra. We have attempted to identify this feature by using the mean values of $V_{1}$ and $V_{3}$ for day 2.34 (see above). The most likely candidate is $\mathrm{O}$ I $3 \mathrm{P}-3 \mathrm{D}^{\circ}$, $5 \mathrm{P}-5 \mathrm{~S}^{\circ}$ at $1.1289,1.1300 \mu \mathrm{m}$ (van Hoof 1999 ); we also note that O I was seen at wavelength $\lambda=7774 \AA$ around maximum light by AD2000.

If this is the case, and the $\mathrm{OI}, \mathrm{H}$ and He are cospatial, then we can make a crude estimate of the $\mathrm{O}$ abun-

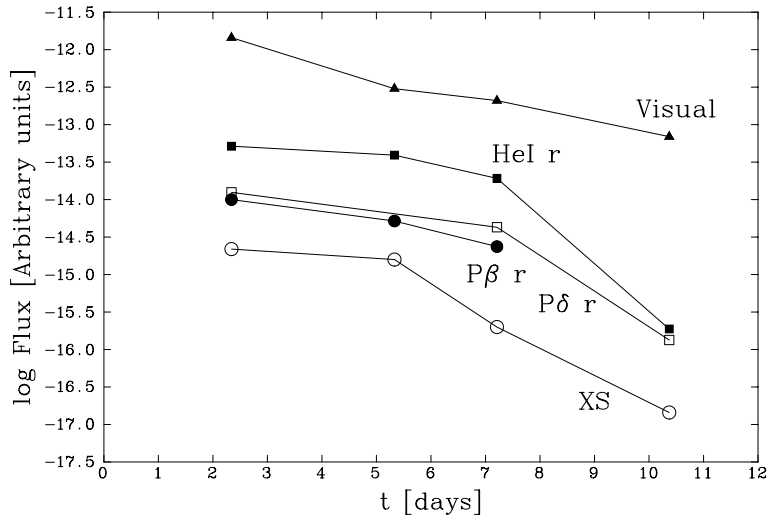

Fig. 7. Dependence of integrated flux in visual flux (filled triangles), the red wings of the He I $\lambda 1.083$ line (filled squares), $\mathrm{P} \beta$ (filled circles), $\mathrm{P} \delta$ (open squares) and the excess discussed in the text (open circles).

dance in the ejected material as follows. The flux ratio $f(\mathrm{O}$ I $) / f(\mathrm{P} \beta) \simeq 0.73$ (see Table 3 ). At the electron temperature and density in the environment of U Sco, oxygen is predominantly singly ionized and we expect the $\mathrm{O}$ I line to arise following recombination of $\mathrm{O}$ II; the required recombination coefficient is given by Nahar (1999). We assume that each recombination gives rise to a $\lambda 1.13$ photon; thus the emission coefficient for the $\mathrm{O}$ г $\lambda 1.13$ line is $\simeq 4.9 \times 10^{-25} N_{\mathrm{e}} N\left(\mathrm{O}^{+}\right) \mathrm{cm}^{-3} \mathrm{~s}^{-1}$ at $2 \times 10^{4} \mathrm{~K}$. We again take the emissivity of $\mathrm{H}$ I and He II from Storey \& Hummer (1995) to find that

$\frac{f(\mathrm{O} \mathrm{I})}{f(\mathrm{P} \beta)}=\frac{49.03 N\left(\mathrm{O}^{+}\right)}{1.16 N\left(\mathrm{H}^{+}\right)+1.01 N\left(\mathrm{He}^{++}\right)}$.

At the $N_{\mathrm{e}}$ and $T_{\mathrm{e}}$ in the ejecta of $\mathrm{U} \mathrm{Sco}, n(\mathrm{He}) / n(\mathrm{H}) \simeq$ $n\left(\mathrm{He}^{+}\right) / n\left(\mathrm{H}^{+}\right)$; taking $n(\mathrm{He}) / n(\mathrm{H}) \simeq 4.5$ (see above), we find that

$\frac{n\left(\mathrm{O}^{+}\right)}{n\left(\mathrm{H}^{+}\right)} \simeq \frac{n(\mathrm{O})}{n(\mathrm{H})} \simeq 0.013 \pm 0.003$

(cf. the solar value $6.6 \times 10^{-4}$ ) where the uncertainty follows only from the uncertainty in the flux ratio (see Sect. 4.2). The ejecta are clearly overabundant in O, indicating the result of TNR on the WD. However as we have considered only recombination (i.e. we have not included other modes of populating the upper level) our conclusion should be regarded as tentative. However our $\mathrm{O} / \mathrm{He}$ ratio $\left(2.9 \times 10^{-3}\right)$ is of the same order as that found $\left(7 \times 10^{-3}\right)$ by Williams et al. (1981).

There is therefore evidence for overabundance of both $\mathrm{He}$ and $\mathrm{O}$ in the ejecta; however confirmation requires more rigorous analysis and is beyond the scope of this paper.

\subsubsection{The excess}

The existence of an excess relative to the WD pseudophotosphere was mentioned in Sect. 4.1. We can rule out 
Table 4. $N_{\mathrm{e}}$, flux ratios He II $7-5 / \mathrm{H} \mathrm{I}, \mathrm{He} / \mathrm{H}$ ratio by number, excess at $2.2 \mu \mathrm{m}$ and ejected mass $M$ for $T_{\mathrm{e}}=2 \times 10^{4} \mathrm{~K}$. See text for details.

\begin{tabular}{lcccc}
\hline$t$ (days): & 2.34 & 5.33 & 7.21 & 10.37 \\
\hline$N_{\mathrm{e}}\left(\mathrm{cm}^{-3}\right)$ & $8.32 \times 10^{10}$ & $2.72 \times 10^{10}$ & $7.90 \times 10^{9}$ & $9.13 \times 10^{8}$ \\
& & & & \\
He II 7-5/H I 7-3: & .095 & .215 & .151 & .212 \\
$y=\mathrm{He} / \mathrm{H}:$ & 5.19 & 5.19 & 1.04 & 0.17 \\
& & & & \\
He II 7-5/H I 5-3: & .091 & .219 & .210 & .633 \\
$y=\mathrm{He} / \mathrm{H:}$ & 6.67 & 12.93 & 3.67 & 1.47 \\
& & & & .012 \\
Excess at $2.2 \mu \mathrm{m}\left(10^{-14} \mathrm{~W} \mathrm{~m}^{-2} \mu \mathrm{m}^{-1}\right)$ & 1.91 & 1.55 & .020 & 1.20 \\
$M\left(10^{-7} M_{\odot}\right)$ & 2.09 & 5.19 & 2.35 & \\
\hline
\end{tabular}

an origin in a circumstellar shell surrounding the U Sco system. Even with negligible mass ejection at outburst (Williams et al. 1981), a pre-existing circumstellar shell would have major consequences on the spectral evolution of U Sco during outburst (see AD2000). We also rule out dust emission for similar reasons.

One possibility is that the excess is due to free-free emission, particularly as the excess at $2.2 \mu \mathrm{m}$ declines with time in a similar way to the $\mathrm{H}$ I recombination and $\mathrm{He} \mathrm{I}$ lines (see Fig. 7). If this is the case we can estimate the mass of emitting gas $M$ from

$$
\begin{aligned}
\frac{M}{M_{\odot}} \simeq & 9.6 \times 10^{-8} f_{14} D_{14}^{2} \frac{A}{Z^{2}} \lambda_{\mu \mathrm{m}}^{2} \\
& \times \exp \left[1.4382 / \lambda_{\mu \mathrm{m}} T_{4}\right] \frac{T_{4}^{1 / 2}}{g N_{10}}
\end{aligned}
$$

if the gas is optically thin. Here $f_{14}$ is the free-free flux density in $10^{-14} \mathrm{~W} \mathrm{~m}^{-2} \mu \mathrm{m}^{-1}, D_{14}$ is the distance in units of $14 \mathrm{kpc}, A$ and $Z$ are respectively the atomic weight and atomic numbers of the ions, $\lambda_{\mu \mathrm{m}}$ is the wavelength in $\mu \mathrm{m}$ and $g$ is the Gaunt factor; $T_{4}$ and $N_{10}$ are respectively the electron temperature in units of $10^{4} \mathrm{~K}$ and electron density in $10^{10} \mathrm{~cm}^{-3}$; we take $A / Z^{2} \simeq 1, g \simeq 1$. The magnitude of the excess is listed in Table 4 (see also Fig. 4), together with the emitting mass as deduced from Eq. (1). Given the uncertainty in the placement of the continuum etc., the agreement between the $M$ values is satisfactory; the mean value is $M=2.7( \pm 0.9) \times 10^{-7} M_{\odot}$, in agreement with previous deductions (Williams et al. 1981; AD2000) that the mass ejected by U Sco during eruption is small.

However a proper determination of the ejected mass in this way must await a more rigorous determination of the WD pseudophotosphere continuum.

\section{Conclusions}

We have obtained IR spectroscopy of the 1999 eruption of $\mathrm{U}$ Sco, and find that the spectrum is dominated by $\mathrm{HI}$, He I and HeII lines and the hot WD.

We conclude that $\mathrm{He}$ is overabundant in the ejecta, with $\mathrm{He} / \mathrm{H} \simeq 4.5 \pm 1.7$ by number, and that $\mathrm{O}$ is also overabundant. There is excess emission with respect to the expected WD pseudophotosphere which is likely due to free-free emission; if this interpretation is correct the ejected mass is $\simeq 2.7( \pm 0.9) \times 10^{-7} M_{\odot}$, consistent with other estimates of the mass ejected by U Sco.

The IR development of the RNe U Sco and RS Oph were very different, in that no coronal emission was seen in U Sco. This probably reflects the different natures of the secondary star and compact component in each case (Webbink et al. 1987). Clearly further IR spectroscopy of $\mathrm{RNe}$ during eruption is highly desirable.

Acknowledgements. We thank ESO for declaring U Sco a Target-of-Opportunity, and the various observers whose scheduled programmes were affected as a result. We thank the referee, Dr Anupama, for helpful comments on an earlier version of this paper; A. E. acknowledges a helpful discussion with Brian Warner. We also gratefully acknowledge the work of M. A. Bautista, S. N. Nahar, M. J. Seaton and D. A. Verner, who supplied the data in the Atomic Line List v2.04 in van Hoof (1999).

\section{References}

Allen, C. W. 1973, Astrophysical Quantities (Athlone Press, London)

Anupama, G. C., \& Dewangan, G. C. 2000, AJ, 119, 1359 (AD2000)

Bailey, J., \& Howarth, I. D. 1979, Journal of the British Astronomical Association, 89, 265

Barlow, M., Brodie, J. P., Brunt, C. C., et al. 1981, MNRAS, 195, 61 (B81)

Evans, A., Callus, C. M., Albinson, J. S., et al. 1988, MNRAS, 234, 755

Greenhouse, M. A., Grasdalen, G. A., Woodward, C. E., et al. 1990, ApJ, 352, 307

Hachisu, I., Kato, M., Kato, T., Matsumoto, K., \& Nomoto, K. 2000, ApJ, 534, 189

Howarth, I. D., \& Bailey, J. 1980, Journal of the British Astronomical Association, 90, 265

Hummer, D. G., \& Storey, P. J. 1998, MNRAS, 297, 1073

Iijima, T. 2001, MNRAS, submitted

Johnston, H. M., \& Kulkarni, S. R. 1992, ApJ, 396, 267 
Kahabka, P., Hartmann, H. W., Parmar, A. N., \& Negueruela, I. 1999, A\&A, 347, L43

Krautter, J., et al. 1984, A\&A, 137, 307

Lépine, S., Shara, M. M., Livio, M., \& Zurek, D. 1999, ApJ, 522, L121 (LSLZ)

Lidman, C., Cuby, J.-C., Hainaut, O., \& Melnik, J. 1999, SOFI User Manual, ESO Document LSO-MAN-ESO-40100-0003

Munari, U., Zwitter, T., Tomov, T., et al. 1999, A\&A, 347, L39 (MZT)

Nahar, S. N. 1999, ApJS, 120, 131

Schaefer, B. E., \& Ringwald, F. A. 1995, ApJ, 447, L45

Schmeer, P. 1999, IAUC, 7113

Starrfield, S., Sparks, W. M., \& Truran, J. W. 1985, ApJ, 291, 136

Starrfield, S., Sparks, W. M., \& Shaviv, G. 1988, ApJ, 325, L35
Storey, P. J., \& Hummer, D. G. 1995, MNRAS, 272, 41

Thoroughgood, T. D., Dhillon, V. S., Littlefair, S. P., Marsh, T. R., \& Smith, D. A. 2001, MNRAS, in press

Truran, J. W., Livio, M., Hayes, J., Starrfield, S., \& Sparks, W. M. 1988, ApJ, 324, 345

Tokunaga, A. T. 1998, in Astrophysical Quantities (Revised), 4th edition

van Hoof, P. 1999, Atomic Line List V2.04, http://www.pa.uky.edu/ peter/atomic/

Warner, B. 1995, Cataclysmic Variable Stars (Cambridge University Press)

Webbink, R. F., Livio, M., Truran, J. W., \& Orio, M. 1987, ApJ, 314, 653

Williams, R. E., Sparks, W. M., Gallagher, J. S., et al. 1981, ApJ, 251, 221 